\title{
Superradiant emission of Smith-Purcell radiation
}

\author{
H. L. Andrews, C. H. Boulware, C. A. Brau, and J. D. Jarvis \\ Department of Physics, Vanderbilt University, Nashville, Tennessee 37235, USA
}

(Received 11 July 2005; published 22 November 2005)

\begin{abstract}
Smith-Purcell (SP) radiation is emitted when an electron passes close to the surface of a metallic grating. The radiation becomes coherent (fluence proportional to the square of the number of electrons) when the electrons are in bunches whose dimensions are smaller than the wavelength of the radiation. This has been observed in experiments in which the electrons are prebunched by an rf linac. The enhancement of the spectral intensity is accompanied by large changes in the angular and spectral distribution of the radiation when the electrons appear in periodic bunches. This is called superradiance. Recently, superradiant SP radiation has been observed from a so-called Smith-Purcell free-electron laser (SP-FEL) in which the electrons are bunched by the lasing process. As in other slow-wave structures, the electron beam in a SP-FEL interacts with an evanescent wave for which the phase velocity matches the electron velocity and amplifies it. The frequency of this wave lies below the range of SP radiation and the wave is not radiated except from the ends of the grating. However, the bunching of the electrons by the interaction with the evanescent wave enhances the ordinary Smith-Purcell radiation and changes the angular and spectral distribution due to superradiant effects. In this article, we introduce a new method for computing the SP radiation in three dimensions, including the effects of finite grating length and superradiance due to periodic electron bunching at an arbitrary frequency. We show that the SP radiation develops spectrally and angularly narrow peaks at the harmonics of the bunching frequency. In rf linacs, where the bunches are widely spaced, several closely spaced harmonics lie under the spectral envelope of the emission from a single electron. In a SP-FEL the harmonics are widely spaced and the SP radiation appears in narrow cones at the SP angles corresponding to the harmonics of the bunching frequency. Finally, we calculate the angular spectral fluence radiated by an electron passing over a lamellar grating of finite length, examine its coherent enhancement in SP-FELs and rf linacs, and compare the results with numerical simulations and available experimental data.
\end{abstract}

DOI: 10.1103/PhysRevSTAB.8.110702

PACS numbers: 07.57.Hm, 41.60.Cr, 84.40.Fe

\section{INTRODUCTION}

There is currently substantial interest in the development of THz sources for applications to biophysics, medical and industrial imaging, nanostructures, and materials science [1]. At the present time, available $\mathrm{THz}$ sources fall into three categories: gas lasers, solid-state devices, and electron-beam driven devices. Optically and electrically pumped molecular gas lasers provide hundreds of lines between 40 and $1000 \mu \mathrm{m}$, but they are inherently not tunable. Solid-state $\mathrm{THz}$ sources include $p$-type germanium lasers, quantum-cascade lasers, and excitation of numerous materials with subpicosecond optical laser pulses. $p$-type Ge lasers can be continuously tunable from 1 to $4 \mathrm{THz}$, but require a large (1-T) external magnetic field, and must be operated at $20 \mathrm{~K}$ [2]. Recently, a quantum-cascade laser has produced up to $2 \mathrm{~mW}$ at 4.4 THz, at temperatures up to $50 \mathrm{~K}$ [3]. Short electromagnetic pulses are also sources of broadband $\mathrm{THz}$ radiation. Subpicosecond pulses can be created by optical rectification of infrared laser pulses [4] or by optically switching the photoconductor in a small diode antenna [5].

Electron-beam driven sources of $\mathrm{THz}$ radiation include backward-wave oscillators (BWOs), synchrotrons, and free-electron lasers (FELs). BWOs are commercially available, compact devices that operate from $30-1000 \mathrm{GHz}$ and produce milliwatts of average power [6,7]. Modern synchrotrons with short electron bunches, such as BESSY II in Berlin [8] and the recirculating linac at Jefferson Laboratory [9], produce broadband radiation out to about $1 \mathrm{THz}$ with tens of watts of average power. Conventional FELs have also been operated in the THz region at several laboratories, with average power as high as hundreds of Watts [10-13]. Coherently enhanced $\mathrm{THz}$ emission from relativistic electrons in an undulator has been observed at ENEA-Frascati [14]. However, synchrotrons and conventional FELs require large facilities.

Another source of radiation in the THz region is SmithPurcell (SP) radiation. SP radiation is emitted when an electron passes close to the surface of a grating, as shown in Fig. 1. The virtual photons of the electron field are scattered by the grating, and the wavelength $\lambda_{S P}$ of the radiation observed at the angle $\theta$ from the direction of the electron beam is

$$
\frac{\lambda_{S P}}{L}=\frac{1}{|p|}\left(\frac{1}{\beta}-\cos \theta\right),
$$

where $L$ is the grating period, $\beta c$ the electron velocity, $c$ the speed of light, and $p$ the order of the reflection from the grating (radiation is observed only on negative orders). The wavelength relation (1) is confirmed by the original experiments of Smith and Purcell [15], and by many experiments 


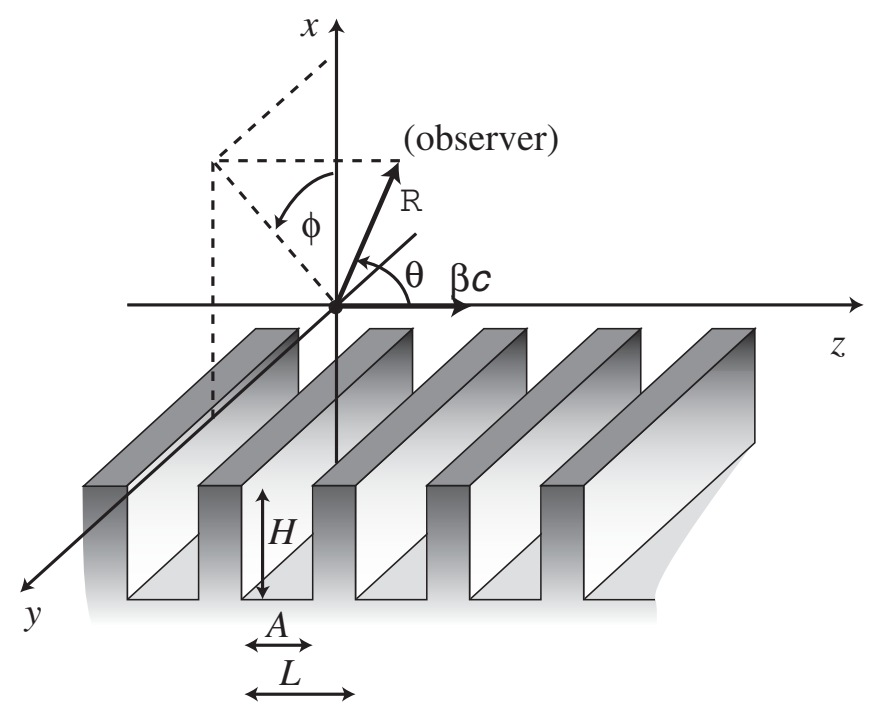

FIG. 1. Smith-Purcell radiation.

conducted since then. Several theories have been advanced to describe the angular fluence of incoherent SP radiation from an infinitely long grating. di Francia used the method of virtual quanta and showed that the interaction of the electron with the grating falls off exponentially with the height of the electron above the grating [16]. van den Berg calculated the radiation from an electron passing over an infinite grating of arbitrary form in two and three dimensions $[17,18]$, and van den Berg and Tan calculated the radiation from an electron passing over a lamellar grating in two dimensions [19]. These theories have been compared with experiments by Woods et al., who also observed coherent enhancement due to bunching of the electrons by the rf linac [20]. Gover et al. used the theory of van den Berg and compared their results to experiments [21]. Shibata et al. extended the work of van den Berg, Haeberle et al. [22], and Schaechter [23] to a lamellar grating of finite length, and discussed its coherent enhancement by individual electron bunches [24]. In addition, they compared the theory with experimental results they obtained using electron beams bunched by rf linacs. Since then, coherent enhancement of SP radiation has become a useful laboratory tool for examining the bunch structure of the micropulses in electron beams [25-27]. Recently, the SP radiation from a finite-length grating of arbitrary profile has been addressed in two dimensions using time- and frequency-domain methods [28], and the coherent radiation from bunches passing over a finite grating of arbitrary profile has been computed in three dimensions using integral-equation methods [29].

A potentially more powerful source of $\mathrm{THz}$ radiation is a tabletop Smith-Purcell free-electron laser (SP-FEL), which consists simply of a grating and an electron beam traveling parallel to the grating surface as indicated in Fig. 1. In addition to scattered waves that propagate to infinity above the grating (SP radiation), there are evanescent modes that travel along the grating and decay exponentially above it. When the phase velocity of the evanescent wave is synchronous with the electron velocity, the electron beam amplifies the evanescent wave $[30,31]$. The group velocity of the evanescent wave can be either positive, as in a traveling-wave tube, or negative, as in a BWO, depending on the dispersive properties of the grating and the velocity of the electrons. When the current in the electron beam exceeds a so-called "start current," the evanescent wave grows to saturation [32]. External feedback is not necessary. A device based on this principle has been demonstrated at Dartmouth $[33,34]$ where nonlinear emission in the direction normal to the grating was observed over the spectral region from $300-900 \mu \mathrm{m}$. The frequency of the evanescent wave lies below the range of SP radiation, so the wave does not radiate except at the ends of the grating. However, bunching of the electrons by the interaction with the evanescent wave enhances the ordinary SP radiation due to superradiant effects [30].

It is well known that when electrons that emit any sort of radiation are bunched into a region small compared to a wavelength, the radiation is strongly enhanced because the electrons emit in phase with one another. In this case, the electromagnetic fields of the electrons add linearly, so the emitted energy increases quadratically with the number of electrons, rather than linearly, but the spectrum is unchanged [35]. This is the hallmark of coherent radiation. When the electron bunches are repeated periodically, the spectral intensity of the emission is enhanced at the bunching frequency and its harmonics, but the total energy emitted per electron remains of the same order of magnitude. This is called superradiance, and it has been discussed previously in the context of synchrotron and undulator radiation [36].

In this article, we address the incoherent, coherent, and superradiant emission of SP radiation. Previous authors, beginning with van den Berg, have computed the angular intensity of SP radiation from a single electron or a random distribution of electrons passing over an infinitely long grating [16-22]. Shibata et al., following van den Berg, calculated the angular intensity of coherent radiation from a single bunch of electrons, and then included a factor to describe the spectral distribution of the radiation from a finite grating [24]. We proceed from a different point of view and introduce a new method for computing SP radiation that incorporates $a b$ initio the effects of finite grating length and multiple electrons. We then use this theory to analyze the enhancement of SP radiation by periodic bunching of the electrons at an arbitrary frequency. The results show that in rf linacs, where the bunches are widely spaced, several closely spaced harmonics lie under the spectral envelope of the emission from a single electron. On the other hand, in an SP-FEL the bunches formed by the interaction with the evanescent wave are closely spaced. As a result, the harmonics are widely spaced and the SP 
radiation appears in spectrally and spatially narrow cones at the SP angles corresponding to the harmonics of the bunching frequency.

The program for carrying out these calculations consists of two parts. In the first part, Sec. II, we develop the relationship between the angular spectral fluence from random electrons passing over a grating of finite length and that from individual and periodic bunches of electrons. In the second part, Sec. III, we calculate the angular spectral fluence from a single electron passing over a finite grating of arbitrary profile. To obtain numerical results, we calculate the angular spectral fluence radiated by electrons passing over a lamellar grating of finite length and examine the coherent enhancement caused by bunching in rf linacs and SP-FELs. Finally, we compare the results with numerical simulations carried out using particle-in-cell codes and with available experimental data.

\section{COHERENT AND SUPERRADIANT EMISSION FROM A FINITE GRATING}

\section{A. Theory}

For a single electron passing over a periodic grating, the angular spectral fluence of radiation at frequency $\omega$ at point $\mathbf{R}=(R, \theta, \phi)$ a large distance from a grating located near the origin, as shown in Fig. 1, is [37]

$$
\frac{d^{2} \mathcal{W}}{d \omega d \Omega}=\frac{2 c R^{2}}{\mu_{0}}|\tilde{\mathbf{B}}(\omega)|^{2},
$$

where $\mu_{0}$ is the permeability of free space (SI units are used throughout) and

$$
\tilde{\mathbf{B}}(\omega)=\frac{1}{\sqrt{2 \pi}} \int_{-\infty}^{\infty} d t e^{i \omega t} \mathbf{B}(t)
$$

is the Fourier transform of the magnetic field $\mathbf{B}(t)$ at point R. But for an electron with the trajectory $z=\beta c t^{\prime}+\delta z$, where $\delta z$ is the position at $t^{\prime}=0$, the radiation emitted when the particle is at point $z$ reaches the observer at the later time

$$
\begin{aligned}
t & =t^{\prime}+\frac{R-z \cos \theta}{c} \\
& =\frac{R-\delta z \cos \theta}{c}+\frac{1-\beta \cos \theta}{\beta} \frac{z-\delta z}{c} .
\end{aligned}
$$

We can use this to change from the variable $t$ to the variable $z$ in (3).

Far from the ends of the grating, the field emitted by the particle must be periodic, and in the far field it must fall off as $1 / R$. Therefore, for a long grating we can ignore the errors at the ends and represent the magnetic field by the expression

$$
\begin{aligned}
\mathbf{B}[t(z)] & =\frac{1}{R} \sum_{p=-\infty}^{\infty} \mathbf{a}_{p}(\hat{\mathbf{R}}) e^{i p K z} & & -\frac{1}{2} Z_{g}<z<\frac{1}{2} Z_{g} \\
& =0 & & \text { otherwise, }
\end{aligned}
$$

where $Z_{g}$ is the length of the grating, $K=2 \pi / L$ the grating wave number, $L$ the grating period, and $p$ is an integer. The vector coefficients $\mathbf{a}_{p}(\hat{\mathbf{R}})$ depend on the unit vector $\hat{\mathbf{R}}=(\theta, \phi)$ pointing to the observer, as well as the electron velocity, height above the grating, and grating profile. By making this approximation, we exclude from consideration several kinds of radiation. The first is edge radiation. This is the radiation emitted when an electron passes over the edge of a smooth block of metal similar to the grating but without grooves. Another form of radiation that we do not consider comes from the evanescent modes of the grating. These modes are excited by the passage of individual electrons or bunches of electrons near the grating [38], and are in fact responsible for oscillation in SPFELs [30-32]. Although they do not radiate directly from the grating, the evanescent waves propagate along the grating. They scatter and reflect when they reach the ends of the grating, and produce radiation at frequencies below those of any SP radiation [31,38,39].

Substituting (5) into (3) and carrying out the integral, we find that

$$
\begin{aligned}
\tilde{\mathbf{B}}(\mathbf{R}, \omega)= & \frac{Z_{g}}{\sqrt{2 \pi}} \frac{1-\beta \cos \theta}{\beta c R} e^{i \frac{\omega}{c}\left(R-\frac{\delta z}{\beta}\right)} \\
& \times \sum_{p=-\infty}^{\infty} \mathbf{a}_{p} \operatorname{sinc}\left[\frac{Z_{g}}{2}\left(\frac{\omega}{c} \frac{1-\beta \cos \theta}{\beta}+p K\right)\right] .
\end{aligned}
$$

The sinc function has peaks at the frequencies $\omega_{p}$ for which

$$
\frac{\omega_{p}}{c} \frac{1-\beta \cos \theta}{\beta}=-p K
$$

This is just another form of the Smith-Purcell relation (1), and we recognize that $p$ is the order of the reflection from the grating. The spectral width of the peaks (at the first zero of the sinc function) is given by the resolution of the grating,

$$
\frac{\delta \omega_{p}}{\omega_{p}}=\frac{1}{|p| N_{g}},
$$

where $N_{g}=Z_{g} / L$ is the number of grooves in the grating. For $N_{g} \gg 1$, the orders are well separated and we can discuss individually the fluence on each order. If we substitute (6) back into (2), we find that the angular spectral fluence on order $p$ for a single electron is

$$
\begin{aligned}
\frac{d^{2} W_{p}^{(1)}}{d \omega d \Omega}= & \frac{Z_{g}^{2}}{\pi \mu_{0} c}\left(\frac{1-\beta \cos \theta}{\beta}\right)^{2}\left|\mathbf{a}_{p}\right|^{2} \\
& \times \operatorname{sinc}^{2}\left[\frac{Z_{g}}{2}\left(\frac{\omega}{c} \frac{1-\beta \cos \theta}{\beta}+p K\right)\right] .
\end{aligned}
$$

If we integrate (9) over all frequencies keeping the direction $\hat{\mathbf{R}}=(\theta, \phi)$ fixed, we find that the angular fluence 
from a single electron is

$$
\frac{d \mathcal{W}_{p}^{(1)}}{d \Omega}=\frac{2 Z_{g}}{\mu_{0}} \frac{1-\beta \cos \theta}{\beta}\left|\mathbf{a}_{p}\right|^{2} .
$$

If we have $N_{e}$ electrons in the beam traveling along the $z$ axis, then the field amplitudes given by (6) add linearly and the total field is

$$
\tilde{\mathbf{B}}^{\left(N_{e}\right)}=\tilde{\mathbf{B}}^{(1)} \sum_{i=1}^{N_{e}} e^{-\left(\omega \delta z_{i} / \beta c\right)},
$$

where $\tilde{\mathbf{B}}^{(1)}$ is the field of a single particle and $\delta z_{i}$ is the position at $t^{\prime}=0$ of the $i$ th particle. The angular spectral fluence on order $p$ is then

$$
\frac{d^{2} \mathcal{W}_{p}^{\left(N_{e}\right)}}{d \omega d \Omega}=\frac{d^{2} \mathcal{W}_{p}^{(1)}}{d \omega d \Omega}\left|\sum_{i=1}^{N_{e}} e^{-i\left(\omega \delta z_{i} / \beta c\right)}\right|^{2} .
$$

For a random distribution of a large number $N_{e} \gg 1$ of electrons with typical spacing $\left|\omega \delta z_{i} / \beta c\right|>O(1)$, the sum is $\approx \sqrt{N}_{e}$, so the total angular spectral fluence is

$$
\frac{d^{2} \mathcal{W}_{p}^{\left(N_{e}\right)}}{d \omega d \Omega}=N_{e} \frac{d^{2} \mathcal{W}_{p}^{(1)}}{d \omega d \Omega}
$$

as is well known. When the electrons form a tight bunch, for which $\left|\omega \delta z_{i} / \beta c\right| \ll O(1)$, the emission becomes coherent. Each term in the sum in unity, so the sum is $\approx N_{e}$ and the angular spectral fluence is

$$
\frac{d^{2} \mathcal{W}_{p}^{\left(N_{e}\right)}}{d \omega d \Omega}=N_{e}^{2} \frac{d^{2} \mathcal{W}_{p}^{(1)}}{d \omega d \Omega} .
$$

The fluence of coherent emission is increased relative to that of incoherent SP radiation from the same number of electrons by the factor $N_{e}$, which can be quite large. However, the spectral and angular distribution is the same. This is well known also, and has been confirmed experimentally [24-27].

More interesting effects occur when the electrons are bunched periodically, as occurs when the electrons interact with the evanescent wave in a SP-FEL. For simplicity we consider the idealized case of $N_{b}$ equally spaced bunches, each with $n_{e}=N_{e} / N_{b}$ electrons. In this case the sum is

$$
\left|\sum_{i=1}^{N_{e}} e^{-i\left(\omega \delta z_{i} / \beta c\right)}\right|=n_{e} \frac{\sin \left(\frac{N_{b}}{2} \frac{\omega z_{b}}{\beta c}\right)}{\sin \left(\frac{1}{2} \frac{\omega z_{b}}{\beta c}\right)},
$$

where $z_{b}$ is the spacing of the bunches. But for $N_{b} \gg 1$, this function has strong, narrow peaks where the denominator vanishes. This happens at the harmonics $\omega_{h}=h \omega_{b}$, where $h$ is an integer and $\omega_{b}=2 \pi \beta c / z_{b}$ is the frequency at which the bunches appear. Typically, in a SP-FEL there are just one or a few harmonics in each order of the SP radiation. Near these peaks we can expand the denominator and get

$$
\left|\sum_{i=1}^{N_{e}} e^{-i\left(\omega \delta z_{i} / \beta c\right)}\right|=N_{e} \operatorname{sinc}\left(\pi N_{b} \frac{\Delta \omega_{h}}{\omega_{b}}\right),
$$

where $\Delta \omega_{h}=\omega-\omega_{h}$. For each harmonic $h$, the angular spectral fluence on order $p$ is then

$$
\begin{aligned}
\frac{d^{2} \mathcal{W}_{p h}^{\left(N_{e}\right)}}{d \omega d \Omega}= & N_{e}^{2} \operatorname{sinc}^{2}\left(\pi N_{b} \frac{\Delta \omega_{h}}{\omega_{b}}\right) \frac{d^{2} \mathcal{W}_{p}^{(1)}}{d \omega d \Omega} \\
= & \frac{N_{e}^{2} Z_{g}^{2}}{\pi \mu_{0} c}\left(\frac{1-\beta \cos \theta}{\beta}\right)^{2}\left|\mathbf{a}_{p}\right|^{2} \\
& \times \operatorname{sinc}^{2}\left(\pi N_{b} \frac{\Delta \omega_{h}}{\omega_{b}}\right) \\
& \times \operatorname{sinc}^{2}\left[\frac{Z_{g}}{2}\left(\frac{\omega}{c} \frac{1-\beta \cos \theta}{\beta}+p K\right)\right] .
\end{aligned}
$$

Relative to the radiation from $N_{e}$ random electrons, the angular spectral fluence at the center of the line is coherently enhanced by the factor $N_{e}$, where $N_{e}$ is the total number of electrons in all the bunches. This enhancement appears in a thin cone at the angle $\theta_{p h}$ that satisfies the Smith-Purcell relation

$$
\cos \theta_{p h}=\frac{1}{\beta}+\frac{p K c}{\omega_{h}} .
$$

Provided that $N_{b} \gg N_{g}$ (which means, in a SP-FEL, that the overall electron beam pulse is much longer than the grating), the first sinc function in (18) is narrower than the second, so the frequency width of the harmonic (at the first zero of the sinc function) is

$$
\frac{\delta \omega_{b}}{\omega_{b}}=\frac{1}{N_{b}} .
$$

For $N_{b} \gg N_{g}$ this is much narrower than the spectral width (8) expected from a single electron or a single small bunch of electrons. This alteration of the angular spectrum relative to that of incoherent or coherent SP radiation is called superradiance [36].

If we integrate (18) over all frequencies, evaluating the second sinc function at $\omega=\omega_{h}$, we find that the angular fluence is

$$
\begin{aligned}
& \frac{d \mathcal{W}_{p h}^{\left(N_{e}\right)}}{d \Omega}=\frac{N_{e}^{2} Z_{g}^{2} \omega_{b}}{\pi \mu_{0} c N_{b}}\left(\frac{1-\beta \cos \theta}{\beta}\right)^{2}\left|\mathbf{a}_{p}\right|^{2} \\
& \quad \times \operatorname{sinc}^{2}\left[\frac{Z_{g}}{2}\left(\frac{\omega_{h}}{c} \frac{1-\beta \cos \theta}{\beta}+p K\right)\right] \\
& =\frac{N_{e} n_{e} N_{g}|p|}{h} \operatorname{sinc}^{2}\left[\frac{Z_{g}}{2}\left(\frac{\omega_{h}}{c} \frac{1-\beta \cos \theta}{\beta}+p K\right)\right] \frac{d \mathcal{W}_{p}^{(1)}}{d \Omega}
\end{aligned}
$$

when we use (19) to evaluate $\cos \theta$ in the slowly varying 
factors. Compared with the radiation from $N_{e}$ random electrons, the superradiant enhancement at line center is $|p| N_{g} n_{e} / h$. This is proportional to the grating resolution and the number of electrons in each bunch, rather than the total number of electrons $N_{e}$. If we expand the argument of the sinc about $\theta_{p h}$, we find that the angular thickness of the cone of harmonic radiation, at the first zero, is

$$
\delta \theta_{p h}=\frac{\lambda_{h}}{Z_{g} \sin \theta_{p h}},
$$

where $\lambda_{h}=2 \pi c / \omega_{h}$ is the wavelength of the harmonic. Subsequent peaks occur at $\Delta \theta_{p h}= \pm \frac{3}{2} \pi, \pm \frac{5}{2} \pi, \ldots$, where $\Delta \theta_{p h}=\theta-\theta_{p h}$ is the angular separation from line center, and fall off in proportion to $\left(\delta \theta_{p h} / \Delta \theta_{p h}\right)^{2}$.

\section{B. Numerical examples}

Coherently enhanced harmonics of the bunching frequency have been observed in recent experiments using an electron beam bunched by an rf linac [40] and in SPFEL experiments $[33,34,41]$. We consider the SP-FEL first. In this case, the electrons enter the region above the grating randomly, and the bunching is induced by the interaction of the electrons with the backward-moving evanescent wave [30-32]. However, this bunching develops quickly if the SP-FEL is strongly saturated, and the error introduced by assuming the electrons are bunched at the beginning of the grating is small. The bunching frequency is determined by the frequency of the evanescent wave, which depends on the dispersive properties of the grating and the electron velocity. For our numerical example we use the parameters of the Dartmouth experiments, which are summarized in Table I [33]. The wavelengths of the first two orders of SP radiation and the first four harmonics of the laser are shown in Fig. 2. At $35 \mathrm{keV}$, for example, the second harmonic of the laser appears near the bottom of the $p=-1$ order at a wavelength $\lambda_{2} \approx 345 \mu \mathrm{m}$ and angle $\theta_{-1,2} \approx 32^{\circ}$. The third harmonic of the laser appears on the $p=-2$ order at the wavelength $\lambda_{3} \approx 230 \mu \mathrm{m}$ and the angle $\theta_{-2,3} \approx 79^{\circ}$. The expected radiation pattern for these experiments is illustrated schematically in Fig. 3, where the evanescent wave is omitted for clarity. Donohue and Gardelle have recently observed similar radiation patterns, including the evanescent wave, in two-dimensional numerical simulations of a SP-FEL using a particle-in-cell code [39].

TABLE I. Parameters of the Dartmouth experiment.

\begin{tabular}{lc}
\hline \hline Grating period & $173 \mu \mathrm{m}$ \\
Groove width & $62 \mu \mathrm{m}$ \\
Groove depth & $100 \mu \mathrm{m}$ \\
Grating length & $12.7 \mathrm{~mm}$ \\
Electron energy & $30-40 \mathrm{keV}$ \\
Electron-beam current & $1 \mathrm{~mA}$ \\
Electron-beam diameter & $24 \mu \mathrm{m}$ \\
\hline \hline
\end{tabular}

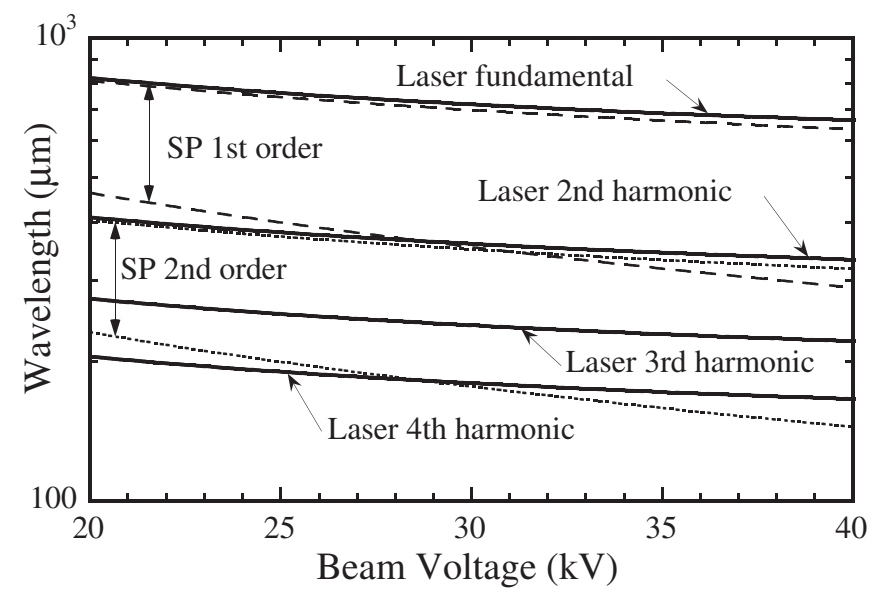

FIG. 2. Wavelengths in the Dartmouth experiments.

Superradiant SP radiation from electron beams bunched by rf linacs represents an opposite limit in which the bunching frequency is very low, so the harmonics are closely spaced rather than widely separated. In general, the number of harmonics that fall under the line width of the SP radiation is

$$
\frac{\delta \omega_{p}}{\omega_{b}}=\frac{\omega_{p}}{N_{g} \omega_{b}}=\frac{\lambda_{b}}{N_{g} \lambda_{p}},
$$

where $\lambda_{p}$ is the wavelength of the radiation and $\lambda_{b}$ the bunch spacing. In the limit when the bunch spacing is large compared to the grating length, many harmonics merge together under the envelope of a single bunch and it is sufficient to consider each bunch individually. Shibata $e t$ $a l$., have analyzed and measured the coherent SP radiation from electrons bunched by an rf linac at RRIKU [24]. The parameters of the experiment are summarized in Table II. In their analysis, Shibata et al. ignore the periodic repeti-

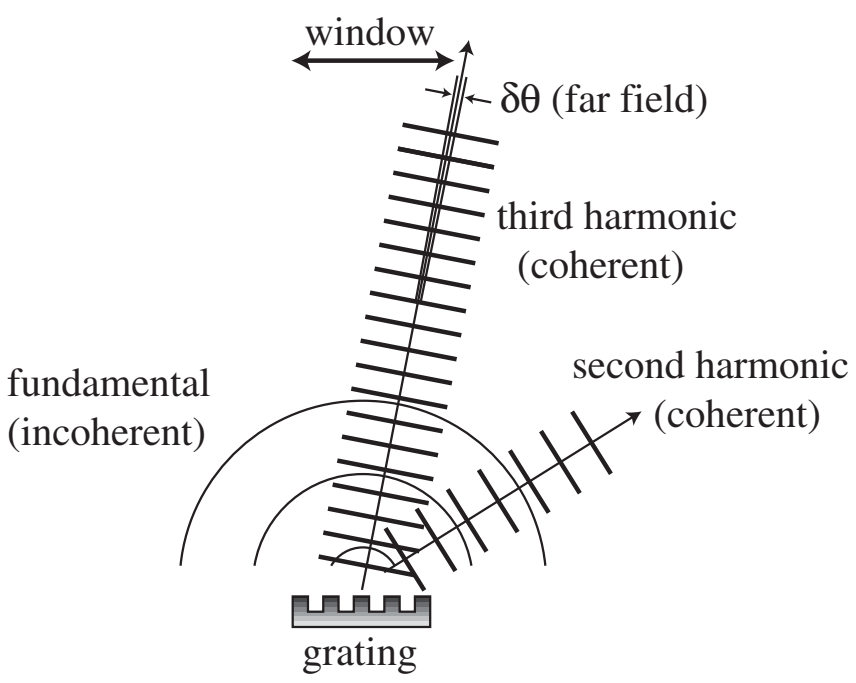

FIG. 3. Radiation pattern in the Dartmouth experiments at $35 \mathrm{keV}$ (not to scale). The evanescent wave is not shown. 
TABLE II. Parameters of the RRIKU experiment..

\begin{tabular}{lc}
\hline \hline Grating period & $6 \mathrm{~mm}$ \\
Groove width & $3 \mathrm{~mm}$ \\
Number of grooves & $20 \mathrm{~mm}$ \\
Wavelength & $3 \mathrm{~mm}$ \\
Electron energy & $40 \mathrm{MeV}$ \\
Bunch spacing & $230 \mathrm{~mm}$ \\
Number of bunches & 43 \\
\hline \hline
\end{tabular}

tion of the bunches at the accelerator frequency and predict only coherent enhancement of the radiation due to a single bunch. However, the harmonics of the bunch frequency were such that about four harmonics would be expected to fall under the line width of the SP emission. Since the number of bunches was larger than the number of grooves in the grating, in these experiments, the width of the harmonics was less than the spacing between them. The harmonics might therefore have been resolvable, but the resolution of the spectrometer was not sufficient for this. However, in more recent experiments on coherent SP radiation from an $\mathrm{rf}$ linac using a double heterodyne receiver, Korbly et al., have resolved the individual harmonics of the bunching frequency [40].

\section{ANGULAR SPECTRAL FLUENCE FROM A SINGLE ELECTRON PASSING OVER A FINITE GRATING}

\section{A. General theory}

To compute the radiation emitted by an electron passing over a grating of a given profile, it is necessary to find the coefficients $\left|\mathbf{a}_{p}\right|^{2}$ that represent the asymptotic form of the magnetic field at the observer far from the grating. To do this, we must solve the Maxwell equations to find the magnetic field $\tilde{\mathbf{B}}$, or at least $|\tilde{\mathbf{B}}|^{2}$, and determine the coefficients by comparison with the asymptotic form (6). The details are explained in the appendix. We summarize the important points here.

Since the field at a distant observer is a plane wave, it is sufficient and convenient to find just the $y$ components (parallel to the grooves, as shown in Fig. 1) $E_{y}$ and $B_{y}$, of the electric and magnetic fields $\mathbf{E}$ and $\mathbf{B}$ rather than the three components of $\mathbf{B}$. The square of the magnetic field is then

$$
c^{2}|\tilde{\mathbf{B}}|^{2}=\frac{\left|\tilde{E}_{y}\right|^{2}+c^{2}\left|\tilde{B}_{y}\right|^{2}}{1-\sin ^{2} \theta \sin ^{2} \phi} .
$$

To solve the Maxwell equations for $\tilde{B}_{y}$, we take the Fourier transform with respect to $y$ (the direction transverse to the beam) and get the two-dimensional Helmholtz equation

$$
\left(\frac{\partial^{2}}{\partial x^{2}}+\frac{\partial^{2}}{\partial z^{2}}\right) \tilde{B}_{k}+\left(\frac{\omega^{2}}{c^{2}}-k_{y}^{2}\right) \tilde{B}_{k}=\frac{\mu_{0} q}{2 \pi} e^{i(\omega z / \beta c)} \frac{\partial}{\partial x} \delta(x),
$$

where $k_{y}$ is the transverse wave number and the subscript $k$ indicates the Fourier transform.

The solution to this equation is the sum of the particular solution and the complementary solution. The particular solution is just the boosted Coulomb field of the electron while the complementary solution is the reflected wave. To take advantage of Floquet's theorem in solving this problem, we consider an infinitely long grating and write the homogeneous solution in the form

$$
\tilde{B}_{k}^{(R)}\left(x, k_{y}, z, \omega\right)=\sum_{p=-\infty}^{\infty} B_{p}\left(k_{y}, \omega\right) e^{i[(\omega / \beta c)+p K] z} e^{i k_{p} x} .
$$

The individual terms (called space harmonics) in (27) are radiative only if $k_{p}=$ real, which requires that

$$
\frac{\omega^{2}}{c^{2}}-\left(\frac{\omega}{\beta c}+p K\right)^{2}>0 .
$$

Since $\beta<1$, the space harmonics are radiative only for $-p_{\max } \leq p \leq-1$, where $p_{\max }$ is some (usually small) integer. The coefficients $B_{p}$ are chosen to satisfy the boundary conditions at the surface of the grating. We leave this tedious part until later.

The magnetic field itself is found by inverting the Fourier transform. At large distances from the grating the Coulomb field can be ignored, so we consider only the reflected field. Inverting the Fourier transform with respect to $y$, we get

$$
\tilde{B}_{y}(\mathbf{R}, \omega)=\frac{1}{\sqrt{2 \pi}} \sum_{p=-p_{\max }}^{-1} \int_{-\infty}^{\infty} d k_{y} B_{p}\left(k_{y}, \omega\right) e^{i\left\{k_{p} x+k_{y} y+[(\omega / \beta c)+p K] z\right\}},
$$

where the sum is over the radiative orders at the frequency $\omega$. We anticipate that $B_{p}\left(k_{y}, \omega\right)$ is a smoothly varying function of $k_{y}$, but for large $R$ the exponential oscillates rapidly except near the stationary-phase point $\bar{k}_{y}$. This point satisfies the condition

$$
\frac{\bar{k}_{y}}{\bar{k}_{p}}=\tan \phi .
$$

That is, the wave vector points towards the observer. The integral can then be evaluated using the method of stationary phase, and we get 


$$
\begin{aligned}
\tilde{B}_{y}(\mathbf{R}, \omega)= & \sum_{p=-p_{\max }}^{-1} e^{i[(\omega / \beta c)+p K] z} e^{i \bar{\psi}_{p}} \bar{B}_{p}(\omega) \\
& \times \sqrt{\frac{\cos ^{2} \phi}{i R \sin \theta} \sqrt{\frac{\omega^{2}}{c^{2}}-\left(\frac{\omega}{\beta c}+p K\right)^{2}}},
\end{aligned}
$$

where

$$
\bar{\psi}_{p}\left(k_{y}\right)=R \sin \theta\left(\bar{k}_{p} \cos \phi+\bar{k}_{y} \sin \phi\right)
$$

and $\bar{B}_{p}$ is the value of $B_{p}$ at the point of stationary phase. The electric field $\tilde{E}_{y}$ is found in a similar manner.

Comparing (31) with (6), we see that $\tilde{B}_{y} \propto 1 / R$ in (6), but that $\tilde{B}_{y} \propto 1 / \sqrt{R}$ in (31). This follows from the fact that in deriving (31) we consider an infinitely long grating, which forms a line source rather than the localized source of a finite-length grating. To connect the coefficients $\mathbf{a}_{p}$ in (6) with the coefficients $B_{p}$ in (31), we must rederive (6) for an infinitely long grating. To do this, we proceed as we did before, except that the distance $\mathbf{r}$ from the electron to the observer depends on the position $z^{\prime}(t)$ of the particle even in the denominator of (5). The Fourier transform is then

$$
\tilde{\mathbf{B}}(\mathbf{R}, \omega)=\frac{1}{\sqrt{2 \pi}} \sum_{p=-p_{\max }}^{-1} \int_{-\infty}^{\infty} d t \frac{\mathbf{a}_{p}(\hat{\mathbf{r}})}{r} e^{i\left[\omega t+p K z^{\prime}(t)\right]} .
$$

We anticipate that $\mathbf{a}_{p}(\hat{\mathbf{r}}) / \mathbf{r}$ is a smoothly varying function of $t$, but the exponential is rapidly oscillating except in the neighborhood of the stationary-phase point $\bar{t}_{p}$, for which

$$
\frac{\omega}{c}\left(\frac{1-\beta \cos \bar{\alpha}_{p}}{\beta}\right)+p K=0,
$$

where $\bar{\alpha}_{p}$ is the angle to the observer from the stationaryphase point. This is just the Smith-Purcell condition, once again. The dominant contribution to the integral comes from the region near the stationary-phase point. That is, while the particle radiates along the entire trajectory from $z^{\prime}=-\infty$ to $z^{\prime}=+\infty$, there is only a small portion of the trajectory for which the Doppler shift puts the radiation at the desired frequency $\omega$. Evaluating the integral in (33) by the method of stationary phase, we get

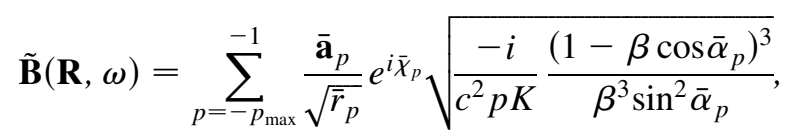

where

$$
\bar{\chi}_{p}=\omega \bar{t}_{p}+p K z^{\prime}\left(\bar{t}_{p}\right)
$$

and $\overline{\mathbf{a}}_{p}$ is the value at the stationary-phase point. Although the integral is dominated by the region around the stationary-phase point, the length of this region increases with the distance of the observer from the surface of the grating. For this reason, the spectral intensity falls off only as fast as the square root of the distance $r_{p}$. For a finite grating, $\bar{\alpha}_{p}=\theta$ and $\bar{r}_{p}=R$, so we are able to make the connection

$$
\begin{aligned}
\left|\mathbf{a}_{p}\right|^{2}= & |p| K \frac{\beta^{3} \sin \theta \cos ^{2} \phi}{(1-\beta \cos \theta)^{3}} \\
& \times \sqrt{\frac{\omega^{2}}{c^{2}}-\left(\frac{\omega}{\beta c}+p K\right)^{2}} \frac{\left|E_{p}\right|^{2}+c^{2}\left|B_{p}\right|^{2}}{1-\sin ^{2} \theta \sin ^{2} \phi} .
\end{aligned}
$$

Provided that the line width of the harmonic is small, we can evaluate the square root at line center using the SmithPurcell relation. The angular spectral fluence is then

$$
\begin{aligned}
\frac{d^{2} W_{p}^{(1)}}{d \omega d \Omega}= & \frac{4 \pi \beta^{2} p^{2} N_{g}^{2}}{\mu_{0} c} \frac{\sin ^{2} \theta \cos ^{2} \phi}{(1-\beta \cos \theta)^{2}} \frac{\left|E_{p}\right|^{2}+c^{2}\left|B_{p}\right|^{2}}{1-\sin ^{2} \theta \sin ^{2} \phi} \\
& \times \operatorname{sinc}^{2}\left[\frac{Z_{g}}{2}\left(\frac{\omega}{c} \frac{1-\beta \cos \theta}{\beta}+p K\right)\right] .
\end{aligned}
$$

If we integrate this expression over all frequencies, we find that the angular fluence is

$$
\frac{d W_{p}^{(1)}}{d \Omega}=\frac{8 \pi^{2} \beta^{3} p^{2} N_{g}^{2}}{Z_{g} \mu_{0}} \frac{\sin ^{2} \theta \cos ^{2} \phi}{(1-\beta \cos \theta)^{3}} \frac{\left|E_{p}\right|^{2}+c^{2}\left|B_{p}\right|^{2}}{1-\sin ^{2} \theta \sin ^{2} \phi} .
$$

It remains to find the coefficients $\left|E_{p}\right|^{2}$ and $\left|B_{p}\right|^{2}$. This must be done for the specific grating profile under consideration.

\section{B. Lamellar gratings}

Up to this point the analysis is independent of the profile of the grating. van den Berg [19] and Haeberle et al. [22] have presented general methods for finding the coefficients $\left|E_{p}\right|^{2}$ and $\left|B_{p}\right|^{2}$ for arbitrary grating profiles. Here we restrict ourselves to the case of a lamellar grating, and adopt the computationally simpler approach used by van den Berg and Tan [19] and by Shibata et al. [24]. In addition to being computationally simple, lamellar gratings are easy to fabricate and have proved to be experimentally convenient and flexible.

As described earlier, the solution to the Helmholtz Eq. (26) is the sum of the complementary solution and the particular solution. The particular solution is just the boosted Coulomb field, and the complementary solution is the reflected field, which is expanded in space harmonics, according to Floquet's theorem. When the solutions are combined to match the boundary conditions on the surface of the grating, we get the matrix equation

$$
\sum_{p=-\infty}^{\infty} M_{p q}^{(B)} B_{q}^{\prime}=V_{p}^{(B)}
$$

for the normalized magnetic field 


$$
B_{p} e^{-i k_{p} H_{e}}=\frac{\mu_{0} q}{4 \pi} e^{i k_{0} H_{e}} B_{p}^{\prime}
$$

where

$$
\begin{gathered}
M_{p q}^{(B)}=\frac{k_{q}}{k_{0}} \delta_{p q}-i 2 \frac{A}{L} \sum_{m=0}^{\infty} \frac{\kappa_{m} \tan \left(\kappa_{m} H\right)}{k_{0}\left(1+\delta_{m 0}\right)} \Psi_{m p} \Psi_{m q}^{*}, \\
V_{p}^{(B)}=-\delta_{p 0}-i 2 \frac{A}{L} \sum_{m=0}^{\infty} \frac{\kappa_{m} \tan \left(\kappa_{m} H\right)}{k_{0}\left(1+\delta_{m 0}\right)} \Psi_{m p} \Psi_{m 0}^{*},
\end{gathered}
$$

and

$$
\Psi_{m q}=\frac{1}{A} \int_{0}^{A} d z e^{-i[(\omega / \beta c)+q K] z} \cos \left(\frac{m \pi z}{A}\right) .
$$

In a similar manner we get the matrix equation

$$
\sum_{p=-\infty}^{\infty} M_{p q}^{(E)} E_{q}^{\prime}=V_{p}^{(E)}
$$

for the normalized electric field

$$
E_{p} e^{-i k_{p} H_{e}}=\frac{\mu_{0} c q}{4 \pi \beta} \frac{k_{y}}{k_{0}} e^{i k_{0} H_{e}} E_{p}^{\prime},
$$

where

$$
\begin{gathered}
M_{p q}^{(E)}=\delta_{p q}-2 i \frac{A}{L} \sum_{m=0}^{\infty} \frac{k_{q}}{\kappa_{m}} \tan \left(\kappa_{m} H\right) \Phi_{m p} \Phi_{m q}^{*}, \\
V_{p}^{(E)}=-\delta_{p 0}-2 i \frac{A}{L} \sum_{m=0}^{\infty} \frac{k_{0}}{\kappa_{m}} \tan \left(\kappa_{m} H\right) \Phi_{m p} \Phi_{m 0}^{*},
\end{gathered}
$$

and

$$
\Phi_{m q}=\frac{1}{A} \int_{0}^{A} d z e^{-i[(\omega / \beta c)+q K] z} \sin \left(\frac{m \pi z}{A}\right) .
$$

The wave numbers satisfy the relations

$$
\begin{gathered}
k_{0}^{2}+k_{y}^{2}=-\frac{\omega^{2}}{\beta^{2} \gamma^{2} c^{2}}, \\
\frac{\omega^{2}}{c^{2}}-\kappa_{m}^{2}-k_{y}^{2}-\left(\frac{m \pi}{A}\right)^{2}=0 .
\end{gathered}
$$

Equations (40) and (45) are easily solved using programs such as MATHCAD. For this purpose it is necessary to truncate the sum over $p$ to $-p_{\text {sum }} \leq p \leq p_{\text {sum }}$. Typically, it is sufficient to use $p_{\text {sum }} \leq 5$.

Substituting these results into (38) and (39) and evaluating $k_{y}$ at the point of stationary phase, we find that in terms of the normalized field coefficients $E_{p}^{\prime}$ and $B^{\prime}{ }_{p}$, the angular spectral fluence on the $p$ th order is

$$
\begin{aligned}
\frac{d^{2} \mathcal{W}_{p}^{(1)}}{d \omega d \Omega}= & \frac{\mu_{0} c q^{2}}{4 \pi} \frac{\beta^{2} p^{2} N_{g}^{2} e^{i 2 k_{0} H_{e}}}{(1-\beta \cos \theta)^{2}} \frac{\sin ^{2} \theta \cos ^{2} \phi}{1-\sin ^{2} \theta \sin ^{2} \phi} \\
& \times\left(\left|\frac{k_{y}}{\beta k_{0}}\right|^{2}\left|E_{p}^{\prime}\right|^{2}+\left|B^{\prime}{ }_{p}\right|^{2}\right) \\
& \times \operatorname{sinc}^{2}\left[\frac{Z_{g}}{2}\left(\frac{\omega}{c} \frac{1-\beta \cos \theta}{\beta}+p K\right)\right]
\end{aligned}
$$

and the angular fluence is

$$
\begin{aligned}
\frac{d \mathcal{W}_{p}^{(1)}}{d \Omega}= & \frac{q^{2}}{2 \varepsilon_{0} L} \frac{\beta^{3} p^{2} N_{g} e^{i 2 k_{0} H_{e}}}{(1-\beta \cos \theta)^{3}} \frac{\sin ^{2} \theta \cos ^{2} \phi}{1-\sin ^{2} \theta \sin ^{2} \phi} \\
& \times\left(\left|\frac{k_{y}}{\beta k_{0}}\right|^{2}\left|E_{p}^{\prime}\right|^{2}+\left|B^{\prime}{ }_{p}\right|^{2}\right),
\end{aligned}
$$

where

$$
\left|\frac{k_{y}}{\beta k_{0}}\right|^{2}=\frac{\gamma^{2} \sin ^{2} \theta \sin ^{2} \phi}{1+\beta^{2} \gamma^{2} \sin ^{2} \theta \sin ^{2} \phi}
$$

and $\gamma=1 / \sqrt{1-\beta^{2}}$ is the relativistic Lorentz factor. Likewise, we see that the exponent is

$$
i 2 k_{0} H_{e}=-\frac{4 \pi|p| H_{e}}{\gamma L(1-\beta \cos \theta)} \sqrt{1+\beta^{2} \gamma^{2} \sin ^{2} \theta \sin ^{2} \phi} .
$$

It should be noted that there are irreconcilable discrepancies in the literature. For the angular fluence, the formula derived by Haeberle et al. [22] differs from (53) by the factor $2 / p^{2}$, but the corresponding formula of Shibata et al. [24] differs by the factor $1 / 4 \pi^{2}$. It is difficult to identify the origin of the discrepancies since these authors derive the corresponding formulas starting with the work of van den Berg [18] and Gover et al. [21], which is different than the approach used here.

\section{Numerical examples}

For a numerical example of these results, we can again use the parameters of the Dartmouth experiments, which are summarized in Table I [33]. The predicted angular distribution of incoherent SP radiation on the first few orders of the grating at a current of $1 \mathrm{~mA}$ is shown in Figs. 4 and 5. The total emission is shown in Fig. 6. We see in Fig. 5 that at low energy the variation of the angular fluence with $\theta$ is smooth. At higher energy $(\mathrm{MeV})$, spikes and singularities are predicted in the angular variation $[22,24]$. The results shown in Fig. 5 for radiation on the fundamental $(p=-1)$ at $\phi=0$ agree closely with those of Urata et al. [33]. As shown in Figs. 4-6, the incoherent emission on the higher orders $p=-2,-3$ is smaller than the first-order emission $p=-1$ by an order of magnitude, and peaks at $\phi \neq 0$.

The start current for SP-FEL oscillation in the Dartmouth experiments is predicted to be about $1 \mathrm{~mA}$, which is in reasonable agreement with the experimental 


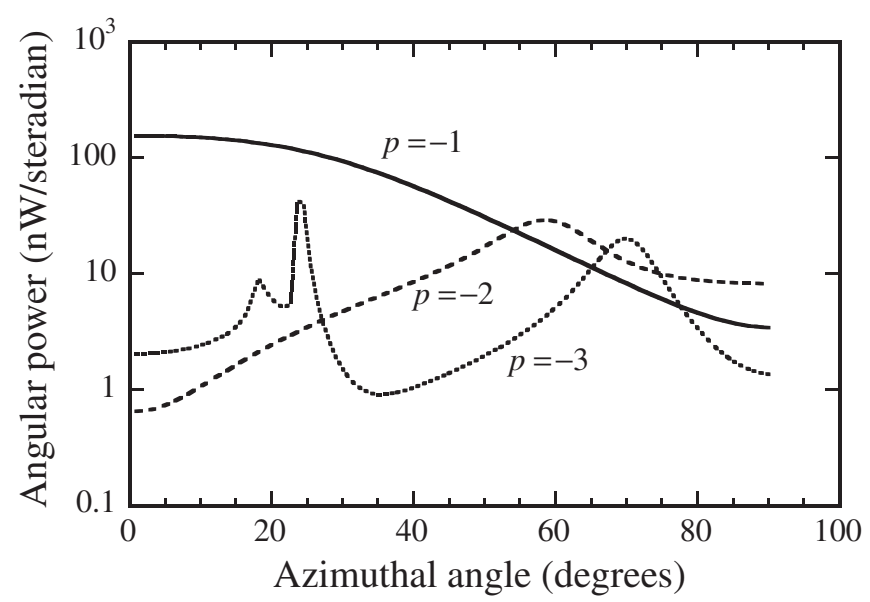

FIG. 4. Angular power of incoherent SP radiation on the first few orders versus azimuthal angle $\phi$ at $\theta=89^{\circ}$.

observation [32]. Below the start current, the observed radiation is dominated by incoherent SP radiation on the $p=-1$ order. Above the start current, bunching of the electrons causes superradiant emission, which increases the intensity and alters the angular spectrum. For the present discussion, it is assumed that the electrons are all in bunches of zero length over the entire grating. While this is certain to be an overestimate, the effect of finite bunch width is not severe for the first few harmonics. Moreover, Donohue and Gardelle observe strong bunching in numerical simulations of SP-FEL dynamics [39]. Figure 6 shows the total power radiated on the second, third, and fourth harmonics, which appear in the first, second, and third orders of SP radiation, respectively. Despite the narrow angular range of superradiant emission, the total superradiant power is orders of magnitude larger than the incoherent emission

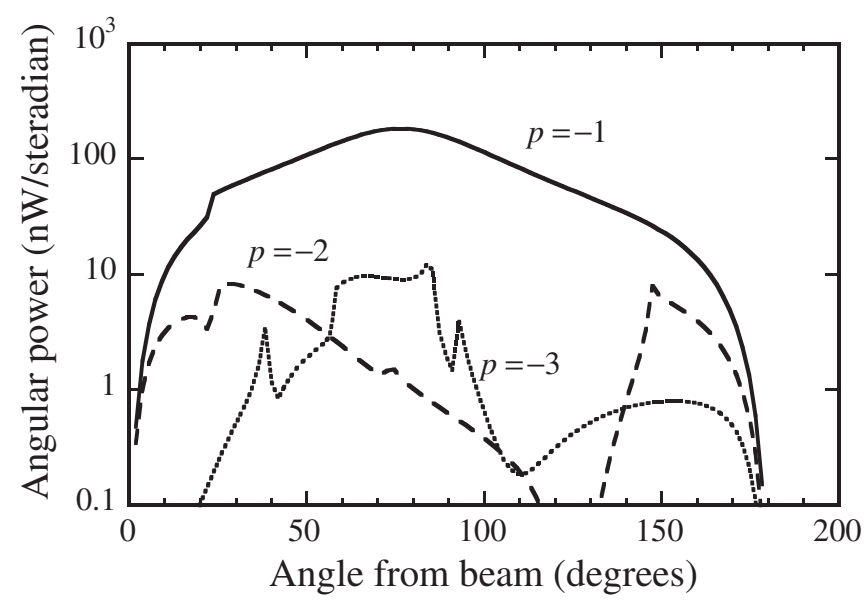

FIG. 5. Angular power of incoherent SP radiation on the first few orders versus angle $\theta$ from the electron beam, at $\phi=0$.

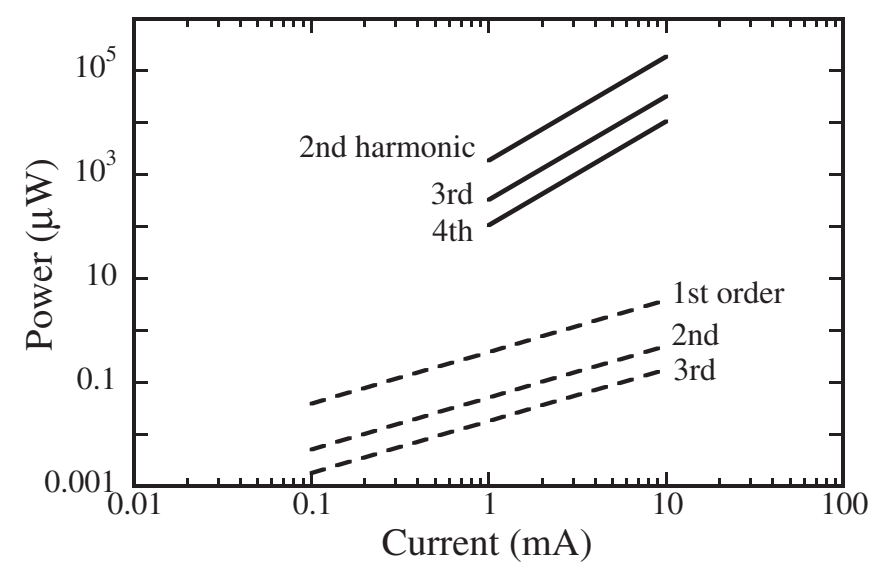

FIG. 6. Total incoherent power radiated on the first, second, and third orders (dotted lines) and total superradiant power radiated on the second, third, and fourth harmonics (solid lines).

\section{CONCLUSIONS}

Using a new theory that includes, $a b$ initio, the effects of periodic electron bunching and finite grating length, we have examined the coherent SP radiation from electron beams bunched by rf linacs and by the evanescent wave in SP-FELs. We have computed the absolute angular spectral fluence of incoherent SP radiation in three dimensions, and the coherent and superradiant enhancement due to periodic bunching of the electron beam.

The results show that for electron beams bunched by rf linacs, in which case the bunch spacing is larger than the length of the grating, the angular spectral fluence observed at low resolution is coherently enhanced by the factor $n_{e}$, the number of electrons in an individual bunch. However, if the number of bunches in a macropulse of the rf linac exceeds the number of grooves in the grating, then several individual harmonics of the rf frequency can be resolved under the envelope of the SP linewidth. These effects are well known and have been observed experimentally [24,40].

Of greater interest are the superradiant effects on SP radiation that occur in SP-FELs. Below the start current for laser oscillation, the radiation is incoherent and appears at all angles with a wavelength given by the Smith-Purcell relation. However, above the start current the radiation pattern becomes much more complex. The laser (evanescent-wave) frequency lies below the lowest order of SP radiation and the evanescent wave does not itself radiate except at the ends of the grating. However, the harmonics of the laser frequency are strongly enhanced in the SP radiation. Since there has been confusion on this point, it should be emphasized that the harmonic radiation observed is superradiant SP radiation from the electrons. It is not radiation scattered from the evanescent wave by the grating. In the absence of the electron beam, the evanescent wave propagates along the surface of the grating without scattering and without loss, aside from dissipative losses in 
the surface of the grating itself [32]. The coherent harmonics appear in distinct patterns having the form of thin cones about the direction of the electron beam at the SP angles corresponding to the harmonic wavelengths. Typically, one harmonic (or at higher energies, a few harmonics) may appear in each order of the SP radiation.

For numerical examples we use the parameters of the Dartmouth SP-FEL experiments, which had an electronbeam current on the order of $1 \mathrm{~mA}$. Numerical simulations using a particle-in-cell code indicate that at saturation the bunching of the electrons by the laser interaction in a SPFEL is very strong [39], and we assume for present purposes that the bunching is perfect when the electron-beam current exceeds the start current for SP-FEL oscillation. Referring to Fig. 6, we see that in the absence of lasing (below the start current), incoherent SP radiation on the first order is predicted to have a total power on the order of $1 \mathrm{~mW}$ at an electron-beam current of $1 \mathrm{~mA}$. The second and third harmonics are about an order of magnitude smaller. However, above the start current the superradiant power on the third harmonic is predicted to be on the order of $100 \mathrm{~mW}$. Thus, while superradiant emission on the coherent harmonics is expected to be weaker than the laser radiation itself, it is much stronger than incoherent SP radiation and it appears at shorter wavelengths than the laser radiation. This will be useful in some applications.

The theoretical predictions of electron bunching at the laser (evanescent-wave) frequency [31,32] are in complete agreement with the numerical simulations of Donohue and Gardelle [39]. Moreover, the angular spectrum of superradiant emission that they observe in the simulations agrees with that predicted by the analytical theory described here. More recently, Li has performed three-dimensional simulations of radiation from a single electron bunch [38]. While the SP radiation from a single bunch in these simulations does not exhibit the superradiant behavior characteristic of periodic bunches, the results show significant excitation of the evanescent wave and scattering of that wave at both ends of the grating. Thus, the theory seems to be in complete agreement with the available numerical simulations.

However, neither the analytical theory nor the numerical simulations completely explain the reported experimental observations $[33,34,42]$. Superradiant enhancement of the emission at normal incidence to the grating is reported for the first three orders of SP radiation [41]. Enhancement of the second-order $(p=-2)$ emission can be explained by the fact that the third harmonic is nearly coincident (within the experimental resolution [42]) with the second-order SP frequency in the direction normal to the grating. Although the third harmonic peaks at $\theta=79^{\circ}$ in the far field, which lies slightly outside the $13^{\circ}$ diameter of the window used in the experiments [42], the near-field radiation is much wider (on the order of the width of the grating), as indicated in Fig. 3, and some fraction of the radiation pattern would be expected to overlap the window. However, superradiant effects, as predicted by the analytic theory and observed in the numerical simulations, cannot explain the reported enhancement of the first-order $(p=-1)$ SP radiation in the direction normal to the grating, where the window was located. Although radiation at the second harmonic of the bunching frequency is emitted on the first order of SP radiation, the emission occurs at an angle about $32^{\circ}$ from the electron beam. This lies well outside the window and, perhaps more importantly, the second-harmonic frequency does not correspond to that of first-order SP radiation in the direction normal to the grating. Moreover, radiation at the laser (bunching) frequency itself has never been observed in the SP-FEL experiments [41], although it is the source of milliwatts of radiation in BWOs [7]. Finally, in the experiments at Dartmouth it was not possible to examine the angular distribution of the superradiant emission. It will be important in future experiments to examine the angular distribution of the superradiant emission and to look for the evanescent wave itself at longer wavelengths [42].

\section{ACKNOWLEDGMENTS}

The authors gratefully acknowledge useful discussions with Hayden Brownell, John Donohue, and Dazhi Li. This work was supported by the Medical Free-Electron Laser Program of the Department of Defense under Grant No. F49620-01-1-0429.

\section{APPENDIX: ANGULAR SPECTRAL FLUENCE FROM A FINITE GRATING}

Since the field at a distant observer is a plane wave, we can find $|\mathbf{B}|^{2}$ from just the $y$ components $E_{y}$ and $B_{y}$ of the electric and magnetic fields. We can see this in the following way. In a plane wave, the electric and magnetic fields are orthogonal to each other and to the unit vector $\hat{\mathbf{R}}$ pointing to the observer. Then, since $|\mathbf{E}|^{2}=c^{2}|\mathbf{B}|^{2}$ in a plane wave, for any direction $\hat{\boldsymbol{\alpha}}$ orthogonal to $\hat{\mathbf{R}}$, we have

$$
\begin{aligned}
|\mathbf{E} \cdot \hat{\boldsymbol{\alpha}}|^{2}+c^{2}|\mathbf{B} \cdot \hat{\boldsymbol{\alpha}}|^{2} & =|\mathbf{E}|^{2} \cos ^{2} \alpha+c^{2}|\mathbf{B}|^{2} \sin ^{2} \alpha \\
& =c^{2}|\mathbf{B}|^{2},
\end{aligned}
$$

where $\alpha$ is the angle between $\mathbf{E}$ and $\hat{\boldsymbol{\alpha}}$. But the vectors $\mathbf{E}$ and $\mathbf{B}$ lie in a plane that is perpendicular to $\hat{\mathbf{R}}$. This plane is, therefore, also perpendicular to the plane of $\hat{\mathbf{R}}$ and $\hat{\mathbf{y}}$, where $\hat{\mathbf{y}}$ is a unit vector along the $y$ axis. If we choose the direction $\hat{a}$ to be the intersection of these planes, then since $\mathbf{E}$ and $\mathbf{B}$ can be resolved into components $E_{\alpha}=\mathbf{E} \cdot \hat{\boldsymbol{\alpha}}$ and $B_{\alpha}=\mathbf{B} \cdot \hat{\boldsymbol{\alpha}}$ parallel to $\hat{\boldsymbol{\alpha}}$ and components orthogonal to $\hat{\mathbf{R}}$ and $\hat{\boldsymbol{\alpha}}$, which are also orthogonal to $\hat{\mathbf{y}}$ (since it lies in the plane of $\hat{\mathbf{R}}$ and $\hat{\boldsymbol{\alpha}}$ ), we can write

$$
\begin{aligned}
& E_{y}=E_{\alpha} \cos \xi, \\
& B_{y}=B_{\alpha} \cos \xi,
\end{aligned}
$$


where $\xi$ is the angle between $\hat{\boldsymbol{\alpha}}$ and $\hat{\mathbf{y}}$. Therefore,

$$
c^{2}|\mathbf{B}|^{2}=\frac{\left|E_{y}\right|^{2}+c^{2}\left|B_{y}\right|^{2}}{\cos ^{2} \xi} .
$$

But the component of $\hat{\mathbf{R}}$ in the direction $\hat{\mathbf{y}}$ is

$$
\hat{\mathbf{R}} \cdot \hat{\mathbf{y}}=\sin \xi=\sin \theta \sin \phi,
$$

so

$$
\cos ^{2} \xi=1-\sin ^{2} \xi=1-\sin ^{2} \theta \sin ^{2} \phi .
$$

Therefore,

$$
c^{2}|\mathbf{B}|^{2}=\frac{\left|E_{y}\right|^{2}+c^{2}\left|B_{y}\right|^{2}}{1-\sin ^{2} \theta \sin ^{2} \phi} .
$$

To find the electric and magnetic fields, we must solve the Maxwell equations or, equivalently, the Helmholtz equations

$$
\begin{gathered}
\nabla^{2} \tilde{\mathbf{B}}+\frac{\omega^{2}}{c^{2}} \tilde{\mathbf{B}}=-\mu_{0} \nabla \times \tilde{\mathbf{J}}, \\
\nabla^{2} \tilde{\mathbf{E}}+\frac{\omega^{2}}{c^{2}} \tilde{\mathbf{E}}=\frac{1}{\varepsilon_{0}} \nabla \tilde{\rho}-i \omega \mu_{0} \tilde{\mathbf{J}},
\end{gathered}
$$

where for a point charge $q$ moving along the $z$ axis with the velocity $\beta c$ the Fourier-transformed charge density and current density are

$$
\begin{array}{r}
\tilde{\rho}(\mathbf{r}, \omega)=\frac{q}{\sqrt{2 \pi} \beta c} \delta(x) \delta(y) e^{i(\omega z / \beta c)}, \\
\tilde{\mathbf{J}}(\mathbf{r}, \omega)=\frac{q}{\sqrt{2 \pi}} \delta(x) \delta(y) e^{i(\omega z / \beta c)} .
\end{array}
$$

To simplify these equations, we follow the approach used by van den Berg [18] and take the Fourier transform with respect to the transverse coordinate $y$ (note the negative sign used here in the exponent of the transform)

$$
\begin{aligned}
& \tilde{E}_{k}\left(x, k_{y}, z, \omega\right)=\frac{1}{\sqrt{2 \pi}} \int_{-\infty}^{\infty} d y e^{-i k_{y} y} \tilde{E}_{y}(\mathbf{r}, \omega), \\
& \tilde{B}_{k}\left(x, k_{y}, z, \omega\right)=\frac{1}{\sqrt{2 \pi}} \int_{-\infty}^{\infty} d y e^{-i k_{y} y} \tilde{B}_{y}(\mathbf{r}, \omega),
\end{aligned}
$$

and get the two-dimensional Helmholtz equations

$$
\left(\frac{\partial^{2}}{\partial x^{2}}+\frac{\partial^{2}}{\partial z^{2}}\right) \tilde{E}_{k}+\left(\frac{\omega^{2}}{c^{2}}-k_{y}^{2}\right) \tilde{E}_{k}=i k_{y} \frac{\mu_{0} c q}{2 \pi \beta} e^{i(\omega z / \beta c)} \delta(x),
$$

$$
\left(\frac{\partial^{2}}{\partial x^{2}}+\frac{\partial^{2}}{\partial z^{2}}\right) \tilde{B}_{k}+\left(\frac{\omega^{2}}{c^{2}}-k_{y}^{2}\right) \tilde{B}_{k}=\frac{\mu_{0} q}{2 \pi} e^{i(\omega z / \beta c)} \frac{\partial}{\partial x} \delta(x) .
$$

The solution to these equations is the sum of the particular solution and the complimentary solution. The particular solution is just the boosted Coulomb field of the electron while the complimentary solution is the reflected wave, so we may write

$$
\begin{aligned}
& \tilde{E}_{k}=\tilde{E}_{k}^{(C)}+\tilde{E}_{k}^{(R)}, \\
& \tilde{B}_{k}=\tilde{B}_{k}^{(C)}+\tilde{B}_{k}^{(R)} .
\end{aligned}
$$

To take advantage of Floquet's theorem, we consider an infinitely long grating and write

$$
\begin{aligned}
& \tilde{E}_{k}^{(R)}\left(x, k_{y}, z, \omega\right)=\sum_{p=-\infty}^{\infty} E_{p}\left(k_{y}, \omega\right) e^{i[(\omega / \beta c)+p K] z} e^{i k_{p} x}, \\
& \tilde{B}_{k}^{(R)}\left(x, k_{y}, z, \omega\right)=\sum_{p=-\infty}^{\infty} B_{p}\left(k_{y}, \omega\right) e^{i[(\omega / \beta c)+p K] z} e^{i k_{p} x} .
\end{aligned}
$$

From the Helmholtz equation we see that

$$
\frac{\omega^{2}}{c^{2}}=k_{p}^{2}+k_{y}^{2}+\left(\frac{\omega}{\beta c}+p K\right)^{2} .
$$

The fields themselves are found by inverting the Fourier transforms. At large distances from the grating we can ignore Coulomb field and consider only the reflected field. Inverting the Fourier transform with respect to $y$, we get

$$
\tilde{B}_{y}(\mathbf{R}, \omega)=\frac{1}{\sqrt{2 \pi}} \sum_{p=-p_{\max }}^{-1} \int_{-\infty}^{\infty} d k_{y} B_{p}\left(k_{y}, \omega\right) e^{i\left\{k_{p} x+k_{y} y+[(\omega / \beta c)+p K] z\right\}},
$$

where the sum is over the radiative orders at the frequency $\omega$. But for a finite grating located near the origin, the coordinates of the observer are

$$
\begin{gathered}
x=R \sin \theta \cos \phi, \\
y=R \sin \theta \sin \phi, \\
z=R \cos \theta,
\end{gathered}
$$


so we can write this in the form

$$
\tilde{B}_{y}(\mathbf{R}, \omega)=\frac{1}{\sqrt{2 \pi}} \sum_{p=-p_{\max }}^{-1} e^{i[(\omega / \beta c)+p K] z} \int_{-\infty}^{\infty} d k_{y} B_{p}\left(k_{y}, \omega\right) e^{i \psi_{p}\left(k_{y}\right)}
$$

where

$$
\psi_{p}\left(k_{y}\right)=R \sin \theta\left(k_{p} \cos \phi+k_{y} \sin \phi\right) .
$$

We anticipate that $B_{p}\left(k_{y}, \omega\right)$ is a smoothly varying function of $k_{y}$, but for large $R$ the exponential oscillates rapidly except near the stationary-phase point $\bar{k}_{y}$. This satisfies the condition

$$
\left.\frac{d \psi_{p}}{d k_{y}}\right|_{\bar{k}_{y}}=R \sin \theta\left(\sin \phi+\left.\cos \phi \frac{d k_{p}}{d k_{y}}\right|_{\bar{k}_{y}}\right)=0
$$

so that

$$
\left.\frac{d k_{p}}{d k_{y}}\right|_{\bar{k}_{y}}=-\tan \phi
$$

This is shown graphically in Fig. 7, from which we see that for radiative orders $p$

$$
\begin{aligned}
& \bar{k}_{y}=\sqrt{\frac{\omega^{2}}{c^{2}}-\left(\frac{\omega}{\beta c}+p K\right)^{2}} \sin \phi, \\
& \bar{k}_{p}=\sqrt{\frac{\omega^{2}}{c^{2}}-\left(\frac{\omega}{\beta c}+p K\right)^{2}} \cos \phi .
\end{aligned}
$$

The individual terms (called space harmonics) in (A18) and (A19) are radiative only if $\bar{k}_{p}=$ real, which requires that

$$
\frac{\omega^{2}}{c^{2}}-\left(\frac{\omega}{\beta c}+p K\right)^{2}>0
$$

Since $\beta<1$, this can happen only for $-p_{\max } \leq p \leq-1$, where $p_{\max }$ is some (usually small) integer. The coefficients $E_{p}$ and $B_{p}$ are chosen to satisfy the boundary conditions at the surface of the grating.

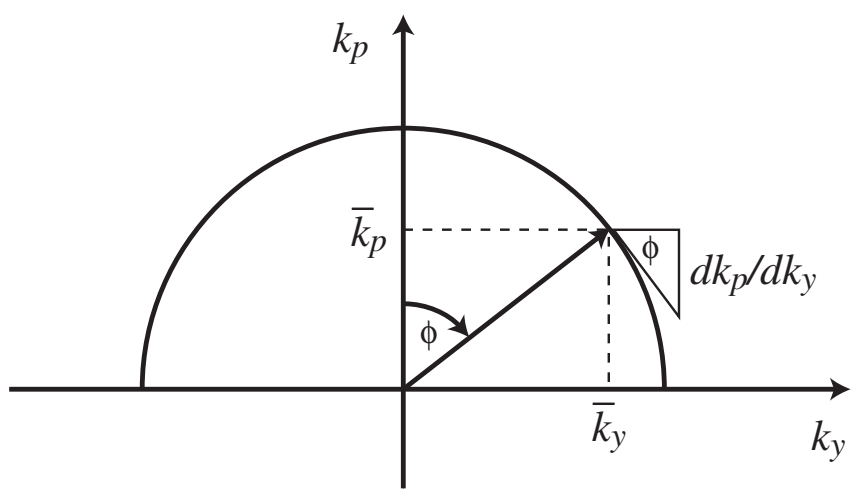

FIG. 7. Graphical representation of wave number relation.
The dominant contribution to the integral (A25) comes from the neighborhood of the point $\bar{k}_{y}$. Using the method of stationary phase, we get

$$
\begin{aligned}
\tilde{B}_{y}(\mathbf{R}, \omega)= & \sum_{p=-p_{\max }}^{-1} e^{i[(\omega / \beta c)+p K] z} e^{i \bar{\psi}_{p}} \bar{B}_{p}(\omega) \\
& \times \sqrt{\frac{\cos ^{2} \phi}{i R \sin \theta} \sqrt{\frac{\omega^{2}}{c^{2}}-\left(\frac{\omega}{\beta c}+p K\right)^{2}}},
\end{aligned}
$$

where $\bar{B}_{p}(\omega)$ and $\bar{\psi}_{p}$ are the values at the stationary-phase point. In the same way, we find that the Fourier transform of the electric field is

$$
\begin{aligned}
\tilde{E}_{y}(\mathbf{R}, \omega)= & \sum_{p=-p_{\max }}^{-1} e^{i[(\omega / \beta c)+p K] z} e^{i \bar{\psi}_{p}} \bar{E}_{p}(\omega) \\
& \times \sqrt{\frac{\cos ^{2} \phi}{i R \sin \theta} \sqrt{\frac{\omega^{2}}{c^{2}}-\left(\frac{\omega}{\beta c}+p K\right)^{2}}} .
\end{aligned}
$$

Comparing (A32) with (6), we see that $\tilde{B}_{y} \propto 1 / R$ in (6), since it applies to the radiation from a localized source (a finite-length grating). On the other hand, $\tilde{B}_{y} \propto 1 / \sqrt{R}$ in (A32) since it applies to the radiation from an infinitely long grating, which forms a line source. To relate the coefficients $\mathbf{a}_{p}$ in (6) to the coefficients $E_{p}$ and $B_{p}$ in (A32) and (A33), we must rederive (6) for an infinitely long grating. Referring to Fig. 8, we argue that the magnetic field at point $\mathbf{R}$ far from the electron is

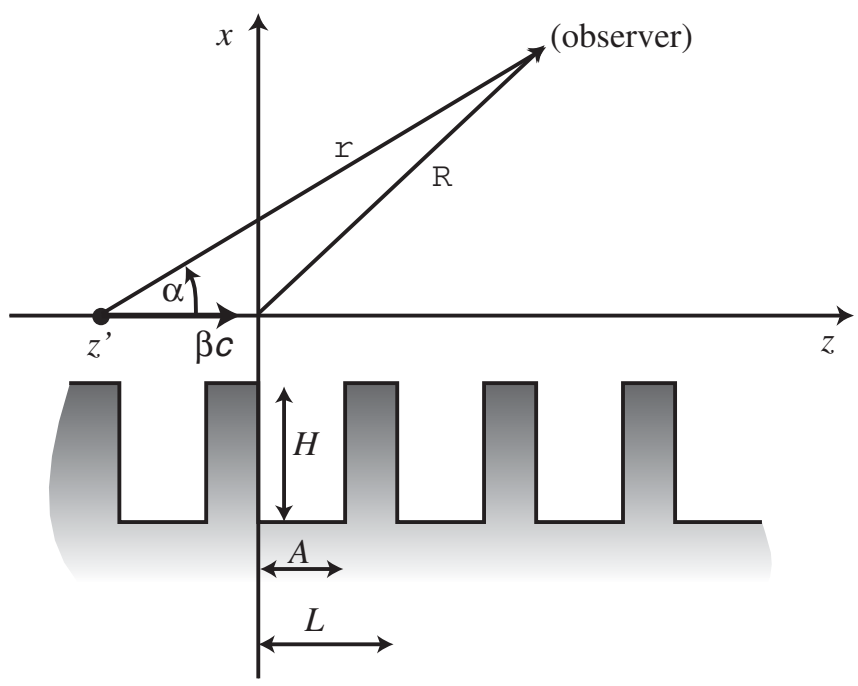

FIG. 8. Radiation from an infinitely long grating. 


$$
\mathbf{B}(\mathbf{R}, t)=\frac{1}{r} \sum_{p=-p_{\max }}^{-1} \mathbf{a}_{p}(\hat{\mathbf{r}}) e^{i p K z^{\prime}}
$$

where $\mathbf{r}$ is the vector from the electron to the observer, and the electron trajectory is

$$
\begin{gathered}
z^{\prime}=\beta c t^{\prime}, \\
t=t^{\prime}+\frac{r}{c}, \\
r^{2}=x^{2}+y^{2}+\left(z-z^{\prime}\right)^{2} .
\end{gathered}
$$

Then,

$$
\tilde{\mathbf{B}}(\mathbf{R}, \omega)=\frac{1}{\sqrt{2 \pi}} \sum_{p=-p_{\max }}^{-1} \int_{-\infty}^{\infty} d t \frac{\mathbf{a}_{p}(\hat{\mathbf{r}})}{r} e^{i \chi_{p}(t)},
$$

where the phase is

$$
\chi_{p}(t)=\omega t+p K z^{\prime}(t)
$$

In (A38), $\mathbf{a}_{p}(\hat{\mathbf{r}}) / \mathbf{r}$ is a smoothly varying function of $t$, but the exponential is rapidly oscillating except in the neighborhood of the stationary-phase point $\bar{t}_{p}$, where

$$
\left.\frac{d \chi_{p}}{d t}\right|_{\bar{t}_{p}}=\omega+\left.p k \frac{d z^{\prime}}{d t}\right|_{\bar{t}_{p}}=0 .
$$

Carrying out the derivatives, we find that the stationaryphase point satisfies the Smith-Purcell condition

$$
\frac{\omega}{c}\left(\frac{1-\beta \cos \bar{\alpha}_{p}}{\beta}\right)+p K=0,
$$

where $\bar{\alpha}_{p}$ is the angle from the stationary-phase point to the observer. The dominant contribution to the integral comes from the region near the stationary-phase point. Evaluating the integral in (A38) by the method of stationary phase, we get

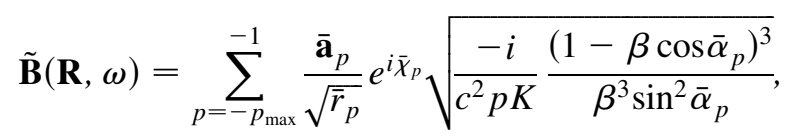

where $\overline{\mathbf{a}}_{p}$ is the value at the stationary-phase point. Provided that the orders are narrow and well separated, so we can use the Smith-Purcell relation and can ignore cross terms, the absolute square of this is

$$
c^{2}|\tilde{\mathbf{B}}(\mathbf{R}, \omega)|^{2}=\sum_{p=-p_{\max }}^{-1} \frac{\left|\overline{\mathbf{a}}_{p}\right|^{2}}{\bar{r}_{p} \mathrm{pK}} \frac{\left(1-\beta \cos \bar{\alpha}_{p}\right)^{3}}{\beta^{3} \sin ^{2} \bar{\alpha}_{p}} .
$$

But from (A7), (A32), and (A33) we see that for narrow, well-separated orders,

$$
\begin{aligned}
c^{2}|\tilde{\mathbf{B}}(\mathbf{R}, \omega)|^{2}= & \sum_{p=-p_{\max }}^{-1} \frac{\left|\bar{E}_{p}\right|^{2}+c^{2}\left|\bar{B}_{p}\right|^{2}}{1-\sin ^{2} \theta \sin ^{2} \phi} \frac{\cos ^{2} \phi}{R \sin \theta} \\
& \times \sqrt{\frac{\omega^{2}}{c^{2}}-\left(\frac{\omega}{\beta c}+p K\right)^{2}} .
\end{aligned}
$$

For a finite grating $\bar{\alpha}_{p}=\theta$ and $\bar{r}_{p}=R$, so by comparing (A43) and (A44) we obtain the relation

$$
\begin{aligned}
\left|\mathbf{a}_{p}\right|^{2}= & |p| K \frac{\beta^{3} \sin \theta \cos ^{2} \phi}{(1-\beta \cos \theta)^{3}} \\
& \times \sqrt{\frac{\omega^{2}}{c^{2}}-\left(\frac{\omega}{\beta c}+p K\right)^{2}} \frac{\left|E_{p}\right|^{2}+c^{2}\left|B_{p}\right|^{2}}{1-\sin ^{2} \theta \sin ^{2} \phi} .
\end{aligned}
$$

The angular spectral fluence from a finite grating is therefore given by the expression

$$
\begin{aligned}
\frac{d^{2} W_{p}^{(1)}}{d \omega d \Omega}= & \frac{\beta|p| K Z_{g}^{2}}{\pi \mu_{0} c} \frac{\sin \theta \cos ^{2} \phi}{1-\beta \cos \theta} \\
& \times \sqrt{\frac{\omega^{2}}{c^{2}}-\left(\frac{\omega}{\beta c}+p K\right)^{2}} \frac{\left|E_{p}\right|^{2}+c^{2}\left|B_{p}\right|^{2}}{1-\sin ^{2} \theta \sin ^{2} \phi} \\
& \times \operatorname{sinc}^{2}\left[\frac{Z_{g}}{2}\left(\frac{\omega}{c} \frac{1-\beta \cos \theta}{\beta}+p K\right)\right] .
\end{aligned}
$$

Provided that the line width is small, we can evaluate the square root at line center using the Smith-Purcell relation and get

$$
\sqrt{\frac{\omega^{2}}{c^{2}}-\left(\frac{\omega}{\beta c}+p K\right)^{2}}=\frac{\beta K|p| \sin \theta}{1-\beta \cos \theta} .
$$

The angular spectral fluence is then

$$
\begin{aligned}
\frac{d^{2} W_{p}^{(1)}}{d \omega d \Omega}= & \frac{4 \pi \beta^{2} p^{2} N_{g}^{2}}{\mu_{0} c} \frac{\sin ^{2} \theta \cos ^{2} \phi}{(1-\beta \cos \theta)^{2}} \frac{\left|E_{p}\right|^{2}+c^{2}\left|B_{p}\right|^{2}}{1-\sin ^{2} \theta \sin ^{2} \phi} \\
& \times \operatorname{sinc}^{2}\left[\frac{Z_{g}}{2}\left(\frac{\omega}{c} \frac{1-\beta \cos \theta}{\beta}+p K\right)\right] .
\end{aligned}
$$

[1] S. P. Mickan and X.-C. Zhang, Int. J. High Speed Electron. Syst. 13, 601 (2003).

[2] E. Brundermann, D. R. Chamberin, and E. E. Haller. Infrared Phys. Technol. 40, 141 (1999).

[3] R. Koehler, A. Tredicucci, F. Beltram, H. E. Beere, E. H. Linfield, A. G. Davies, D. A. Ritchie, R. C. Iotti, and F. Rossi, Nature (London) 417, 156 (2002).

[4] A. Bonvalet and M. Joffre, Terahertz Femtosecond Pulses, in Femtosecond Laser Pulses, edited by C. Rulliere (Springer Verlag, Berlin, 1998).

[5] X.-C. Zhang and D.H. Auston, J. Appl. Phys. 71, 326 (1992).

[6] J.R. Pierce, Traveling-Wave Tubes (D. Van Nostrand Company, New York, 1950).

[7] A. Dobroiu, M. Yamashita, Y. Ohshima, Y. Morita, C. Otani, and K. Kawasi, Appl. Opt. 43, 5637 (2004). 
[8] M. Abo-Bakr, J. Feikes, K. Holldack, G. Wuestefeld, and H.-W. Huebers, Phys. Lett., 88, 254801 (2002).

[9] G. P. Williams, Rev. Sci. Instrum. 73, 1461 (2002).

[10] G. Ramian, Nucl. Instrum. Methods Phys. Res., Sect. A 318, 225 (1992).

[11] N.A. Vinokurov, V.P. Bolotin, D. A. Kayran, B. A. Knyazev, E. I. Kolobanov, V. V. Kotenkov, V. V. Kubarev, G. N. Kulipanov, A. N. Matveenko, L. E. Medvedev, S. V. Miginsky, L. A. Mironenko, A. D. Oreshkov, V. K. Ovchar, V. M. Popik, T. V. Salikova, M.A. Scheglov, S.S. Serednyakov, O.A. Shevchenko, and A. N. Skrinsky, in Proceedings of the International FEL Conference, Trieste, 2004, http://accelconf.web.cern.ch/accelconf/f04/ index.html.

[12] H. Koike, M. Sobajima, V. M. Popik, M. Yokoyama, F. Oda, M. Kawai, K. Toyoda, H. Kuroda, and K. Nakai, Nucl. Instrum. Methods Phys. Res., Sect. A 507, 242 (2003).

[13] Y. U. Jeong, G. M. Kazakevitch, B. C. Lee, S. O. Cho, J. Yoo, N. G. Gavrilov, and V. V. Kubarev, Nucl. Instrum. Methods Phys. Res., Sect. A 507, 125 (2003).

[14] A. Doria, G. P. Gallerano, E. Giovenale, G. Messina, and I. Spassovsky, Phys. Rev. Lett. 93, 264801 (2004).

[15] S. J. Smith and E. M. Purcell, Phys. Rev. 92, 1069 (1953).

[16] G. T. di Francia, Nuovo Cimento 16, 61 (1960).

[17] P. M. van den Berg, J. Opt. Soc. Am. 63, 689 (1973).

[18] P. M. van den Berg, J. Opt. Soc. Am. 63, 1588 (1973).

[19] P. M. van den Berg and T. H. Tan, J. Opt. Soc. Am. 64, 325 (1974).

[20] K. J. Woods, J.E. Walsh, R. E. Stoner, H. G. Kirk, and R. C. Fernow, Phys. Rev. Lett. 74, 3808 (1995).

[21] A. Gover, P. Dvorkis, and U. Elisha, J. Opt. Soc. Am. B 1, 723 (1984).

[22] O. Haeberle, P. Rullhusen, J.-M. Salome, and N. Maene, Phys. Rev. E 49, 3340 (1994).

[23] L. Schaechter, Beam-Wave Interaction in Periodic and Quasi-Periodic Structures (Springer-Verlag, Berlin, 1996).

[24] Y. Shibata, S. Hasebe, K. Ishi, S. Ono, M. Ikezawa, T. Nakazato, M. Oyamada, S. Urasawa, T. Takahashi, T. Matsuyama, K. Kobayashi, and Y. Fujita, Phys. Rev. E 57, 1061 (1998).
[25] G. Doucas, M. F. Kimmitt, A. Doria, G. P. Gallerano, E. Giovenale G. Messina, H. L. Andrews and J. H. Brownell, in Proceedings of the European Particle Accelerator Conference, 2002 (EPS, Geneva, 2002), p. 1870.

[26] G. Doucas, M. F. Kimmitt, A. Doria, G.P. Gallerano, E. Giovenale, G. Messina, H.L. Andrews, and J.H. Brownell, Phys. Rev. ST Accel. Beams 5, 072802 (2002).

[27] A. Doria, G. P. Gallerano, E. Giovenale, G. Messina, G. Doucas, M.F. Kimmitt, H.L. Andrews, and J.H. Brownell, Nucl. Instrum. Methods Phys. Res., Sect. A 483, 263 (2002).

[28] A. S. Kesar, M. Hess, S. E. Korbly, and R. J. Temkin, Phys. Rev. E 71, 016501 (2005).

[29] A. S. Kesar, Phys. Rev. ST Accel. Beams 8, 072801 (2005).

[30] C. S. Liu and V. K. Tripathi, IEEE J. Quantum Electron. 35, 1386 (1999).

[31] H. L. Andrews and C.A. Brau, Phys. Rev. ST Accel. Beams 7, 070701 (2004).

[32] H. L. Andrews, C. H. Boulware, C.A. Brau, and J.D. Jarvis, Phys. Rev. ST Accel. Beams 8, 050703 (2005).

[33] J. Urata, M. Goldstein, M.F. Kimmitt, A. Naumov, C. Platt, and J. E. Walsh, Phys. Rev. Lett. 80, 516 (1998).

[34] A. Bakhtyari, J. E. Walsh, and J. H. Brownell, Phys. Rev. E 65, 066503 (2002).

[35] C. A. Brau, Modern Problems in Classical Electrodynamics (Oxford University Press, New York, 2004), p. 532.

[36] A. Gover, Phys. Rev. ST Accel. Beams 8, 030701 (2005).

[37] C. A. Brau, Modern Problems in Classical Electrodynamics (Oxford University Press, New York, 2004), p. 490.

[38] D. Li, K. Imasaki, G.-S. Park, and Z. Yang, in Proceedings of the 27th International Free Electron Laser Conference, Stanford University, 2005, http://oraweb.cern.ch:9000/pls/ fel05/JACoW.view_abstract?abs_id=1304

[39] J. T. Donohue and J. Gardelle, Phys. Rev. ST Accel. Beams 8, 060702 (2005).

[40] S. E. Korbly, A. S. Kesar, J. R. Sirigiri, and R. J. Temkin, Phys. Rev. Lett. 94, 054803 (2005).

[41] J.H. Brownell (private communication).

[42] J. M. Goldstein et al., Appl. Phys. Lett. 71, 452 (1997). 